\title{
Minimum Average-Cost Path for Real Time 3D Coronary Artery Segmentation of CT Images
}

\author{
Ning Zhu and Albert C.S. Chung \\ Lo Kwee-Seong Medical Image Analysis Laboratory, \\ Department of Computer Science and Engineering, \\ Hong Kong University of Science and Technology, Hong Kong \\ \{nzhu, achung\}@cse.ust.hk
}

\begin{abstract}
In this paper, we propose a Minimum Average-cost Path (MACP) model for segmenting 3D coronary arteries by minimizing the average edge cost along path in discrete $4 \mathrm{D}$ graph constructed by image voxels and associated radii. Prim's Minimum Spanning Tree method is used for efficient optimization of the MACP model. The centerline and the radii of the cross sections of the coronary artery are extracted simultaneously during the optimization. The method does not need any image preprocessing steps and has been intensively validated as an effective approach with the Rotterdam Coronary Artery Algorithm Evaluation Framework [1. The computational cost of the proposed method is particularly low (7.467 seconds per segment, $18.5 \mathrm{~mm} / \mathrm{s}$ on average), which makes real time segmentation of coronary artery possible. Shortcut problem, which is a classic issue of the minimal path techniques, can also be overcome by the proposed method.
\end{abstract}

\section{Introduction}

In recent years, coronary artery segmentation has become an important research issue since it can help diagnosis and treatment of coronary diseases, which is one of the major causes of death around the world. As described in [4, a variety of methods have been proposed for vessel segmentation with the aim of extracting centerlines in 3D space by using classical minimal path algorithms. Some exceptions can be found in 5/711] and they share the same idea of incorporating the cross sectional radius as an additional dimension together with the coordinates of the voxels. Thus when the tracking procedure ends, the radii of the vessel is also obtained. The resultant paths are better centered by using these methods.

Similar to 51711, our approach represents the coronary artery centerline with its radii as a $4 \mathrm{D}$ tree branch with $3 \mathrm{D}$ coordinates of the centerline point and one addition dimension for associated radii. Given the start point, the end point and a point inside the distal part of an artery, our method can trace the centerline with associated radii efficiently without taking the risk of tracing a shortcut path, which is a classical issue of minimal path techniques. Two kinds of shortcut problem are shown in Fig. 11 In extreme case, as shown in Fig. 1(a) the path may be traced outside the vessels (dashed line), while in other shortcut 
cases (Fig. 1(b)], the path (dashed line) obtained by minimal path techniques may stay in the vessels but are less satisfactory than the real centerline path (solid line). The model proposed in this paper, namely Minimum Average-cost Path (MACP), concentrates on low average edge cost by minimizing the newly designed average cost of all edges on the path in the form of probability.

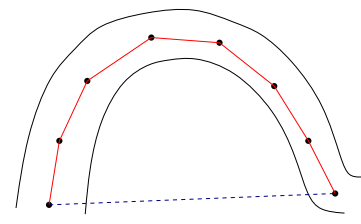

(a) Shortcut Problem 1

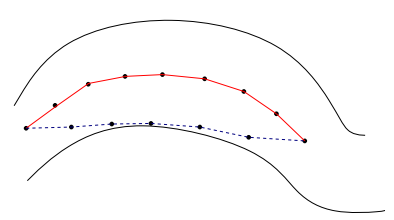

(b) Shortcut Problem 2

Fig. 1. Shortcut Problems of Minimal Path Approaches

To the best of our knowledge, there is only one work [9] on real time 3D CT coronary artery segmentation, but it achieves relatively low accuracy. The MACP model proposed in our paper introduces a new energy term for fast propagation and the proposed method tends to consider much less candidate voxels than minimal path methods. These properties of MACP make it possible to trace the coronary artery with a very low computational cost.

There are four favorable properties of the proposed method. First, according to the experimental results listed in the Rotterdam Coronary Artery Algorithm Evaluation Framework [1, our method is computationally more efficient than all other methods for segmenting 3D CT images of coronary artery. The second merit is that the classical issue of minimal path techniques, shortcut problem, can be overcome by the proposed method. Third, the proposed method does not need any preprocessing steps such as enhancement, and has been validated intensively in the experiments.

\section{Minimum Average-Cost Path Model}

Similar to [5/711, the cross sections of coronary arteries are assumed to be circular as an approximation which is reasonable for thin vessels such as coronaries. The $4 \mathrm{D}$ graph node $X_{t}=\left(\boldsymbol{p}_{t}, r_{t}\right)$ (Fig. (2)), in the assumption includes the voxel position $\boldsymbol{p}_{t}=\left(x_{t}, y_{t}, z_{t}\right)$ and the radii $r_{t}$ of the cross section. We let $\boldsymbol{d}_{t}$ be the direction from the previous node position $\boldsymbol{p}_{t-1}=\left(x_{t-1}, y_{t-1}, z_{t-1}\right)$ to the node position $\boldsymbol{p}_{t}=\left(x_{t}, y_{t}, z_{t}\right)$ being considered. The cross section is defined to be perpendicular to $\boldsymbol{d}_{t}$. Lesage et. al. proposed a Bayesian minimal path method

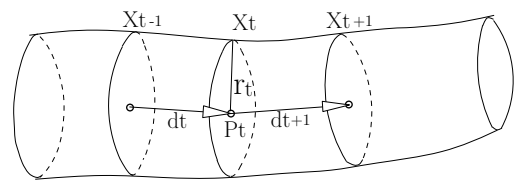

Fig. 2. Geometric Model 
for coronary artery segmentation in [5]. The recursive update of the a posterior probability of a model realization is given by the Bayes' rule:

$$
P\left(X_{[0: t]} \mid Y_{[0: t]}\right) \propto P\left(X_{[0: t-1]} \mid Y_{[0: t-1]}\right) P\left(X_{t} \mid X_{t-1}\right) \frac{P_{v}\left(Y_{t}\right)}{P_{b g}\left(Y_{t}\right)},
$$

where $Y_{t}$ is the observation of $X_{t}, P_{v}\left(Y_{t}\right)$ and $P_{b g}\left(Y_{t}\right)$ are the probabilities of the response as a path node and background node respectively. $P\left(X_{[0: t]} \mid Y_{[0: t]}\right)$ denotes the joint distribution of sequence of nodes on the path given the observations associated with the nodes. Maximizing Equation (1) is equivalent to minimizing its negative logarithm:

$$
\mathrm{C}\left(X_{[0: t]}\right)=\mathrm{C}\left(X_{[0: t-1]}\right)-\log \left(P\left(X_{t} \mid X_{t-1}\right)\right)-\log \left(P_{v}\left(Y_{t}\right)\right)+\log \left(P_{b g}\left(Y_{t}\right)\right)+M,
$$

where $\mathrm{C}\left(X_{[0: t]}\right)=-\log P\left(X_{[0: t]} \mid Y_{[0: t]}\right), X_{t}=\left(\boldsymbol{p}_{t}, r_{t}\right)$ and $M$ is a constant ensuring that $\mathrm{C}\left(X_{[0: t]}\right)$ is positive.

As described in Section [1, the minimal path method in [5] may trace a shortcut path. The shortcut problem is caused by the assumption that the path can be obtained by maximizing $P\left(X_{[0: t]} \mid Y_{[0: t]}\right)$, which is equivalent to minimizing $\mathrm{C}\left(X_{[0: t]}\right)$. This may lead to the problem of shortcut since the value of $\mathrm{C}\left(X_{[0: t]}\right)$ may still become smaller if fewer points are included into the path.

In order to ensure a low average cost path, the value of $P\left(X_{t} \mid Y_{t}, X_{[0: t-1]}\right)$ should be large for each $X_{t}$ in the route. We can assume $P\left(X_{t} \mid Y_{t}, X_{[0: t-1]}\right)=$ $P\left(X_{t} \mid Y_{t}, X_{t-1}\right)$ by regarding the $4 \mathrm{D}$ path as first order Markov chain. Maximizing $P\left(X_{t} \mid Y_{t}, X_{[0: t-1]}\right)$ is equal to minimizing $-\log P\left(X_{t} \mid Y_{t}, X_{t-1}\right)$. As such, we define a new notation ACP in our model:

$$
\begin{aligned}
\operatorname{ACP}(\boldsymbol{v}) & =-\frac{\sum_{j=1}^{t} \log \left\{P\left(X_{j} \mid Y_{j}, X_{j-1}\right)\right\}}{t} \\
& =-\frac{\sum_{j=1}^{t}\left\{\log \left\{P\left(X_{[0: j]} \mid Y_{[0: j]}\right)\right\}-\log \left\{P\left(X_{[0: j-1]} \mid Y_{[0: j-1]}\right)\right\}\right\}}{t} .
\end{aligned}
$$

$\boldsymbol{v}$ here denotes the whole path from the start point $X_{0}$ to the end point $X_{t}$. Considering a single path $\boldsymbol{v}$, our model aims at minimizing $\operatorname{ACP}(\boldsymbol{v})$. Suppose $\mathrm{C}\left(X_{[0: j]}\right)=-\log \left(P\left(X_{[0: j]} \mid Y_{[0: j]}\right)=\mathrm{C}\left(X_{[0: j-1]}\right)+\operatorname{Edge}(j)\right.$,

$$
\operatorname{ACP}(\boldsymbol{v})=\frac{\sum_{j=1}^{t}\left\{\mathrm{C}\left(X_{[0: j]}\right)-\mathrm{C}\left(X_{[0: j-1]}\right)\right\}}{t}=\frac{\sum_{j=1}^{t} \operatorname{Edge}(j)}{t}=\frac{\mathrm{C}\left(X_{[0: t]}\right)-\mathrm{C}\left(X_{0}\right)}{t} .
$$

According to Equation (2), we can obtain

$$
\operatorname{Edge}(j)=-\log \left(P\left(X_{j} \mid X_{j-1}\right)\right)-\log \left(P_{v}\left(Y_{j}\right)\right)+\log \left(P_{b g}\left(Y_{j}\right)\right)+M .
$$

In these steps above, we translate our intuition to the model MACP.

\section{Bayesian Minimum Spanning Tree Implementation}

\subsection{A Novel Energy Term for Fast Propagation}

In this section, an energy term $\mathrm{E}(\boldsymbol{v})$ is defined for fast propagation and optimization of the MACP model. $\boldsymbol{v}$ here denotes the whole path from the start 
point $S$ to the end point $E$.

$$
\mathrm{E}(\boldsymbol{v})=\mathrm{L}(\boldsymbol{v}) / N+\mathrm{ACP}(\boldsymbol{v}),
$$

where $N$ is the total number of nodes on the path except $\mathrm{S}$, and

$$
\begin{aligned}
\mathrm{L}(\boldsymbol{v}) & =-2 \times \operatorname{Dis}(S, E) \\
& =\sum_{i=1}^{N}\left\{\left(\left(\operatorname{Dis}\left(p_{i-1}, S\right)-\operatorname{Dis}\left(p_{i}, S\right)\right)+\left(\operatorname{Dis}\left(p_{i}, E\right)-\operatorname{Dis}\left(p_{i-1}, E\right)\right)\right\},\right.
\end{aligned}
$$

in which $p_{0}=S$ and $p_{N}=E$, and $\operatorname{Dis}(\cdot, \cdot)$ denotes the distance between two points. Assume

$$
\operatorname{LES}(i)=\operatorname{LS}(i)+\operatorname{LE}(i)=\operatorname{Dis}\left(p_{i-1}, S\right)-\operatorname{Dis}\left(p_{i}, S\right)+\operatorname{Dis}\left(p_{i}, E\right)-\operatorname{Dis}\left(p_{i-1}, E\right),
$$

we can obtain,

$$
\mathrm{E}(\boldsymbol{v})=\sum_{i=1}^{N} \operatorname{LES}(i)+\operatorname{Edge}(i) / N
$$

Since $\mathrm{L}(\boldsymbol{v})$ is a negative constant, minimizing $\mathrm{L}(\boldsymbol{v}) / N$ is equal to minimizing $N$. By minimizing $\mathrm{E}(\boldsymbol{v}), \mathrm{ACP}$ is minimized with the constrain of reducing the total number of points on the path. In each propagation phase, we choose the point with the minimum average edge cost from the start point to it according to Equation (4). $\boldsymbol{v}_{j}$ here denotes the path from the start point to the point being considered and the number of points on the path is $j$ :

$$
\mathrm{E}\left(\boldsymbol{v}_{j}\right)=\sum_{i=1}^{j}\{\operatorname{LES}(i)+\operatorname{Edge}(i)\} / j .
$$

\subsection{Implementation Detail}

We modify the Prim's algorithm to fit the proposed model. The overall algorithm is described in Algorithm 1 .

In the experiments, one artery is traced in two parts given the start point $S_{p}$, the end point $E_{p}$ and a point inside the distal part of the artery, $A_{p}$. First, we set $S=A_{p}, E=S_{p}$. With Algorithm 1, the path from $A_{p}$ to $S_{p}$ is obtained. Then we set $S=A_{p}, E=E_{p}$ to obtain the path from $E_{p}$ to $A_{p}$. After that, the whole artery can be obtained by simply connecting the two parts together. The reason for not just tracing back from $E_{p}$ to $S_{p}$ is that the shape of the distal parts, especially the thin ones, are irregular. After step 13 in Algorithm 1, a tree is obtained. And then a low average cost path can be obtained by tracing back from $E$ to $S$.

The proposed method tends to consider much less candidate voxels than the minimal path methods. For example, in Fig. 3. the path from node $A$ to node $T$ is the real path. Different with minimal path methods, the proposed method tends to dismiss the nodes such as $F_{i}(i=1, \ldots n)$ since the average cost from A to $F_{i}$ is large, and does not have to bother the paths to useless nodes $D_{i}(i=1, \ldots n)$ and $E_{i}(i=1, \ldots n)$. For minimal path method, the number of the useless nodes kept in the memory will increase exponentially while propagating towards the end point 


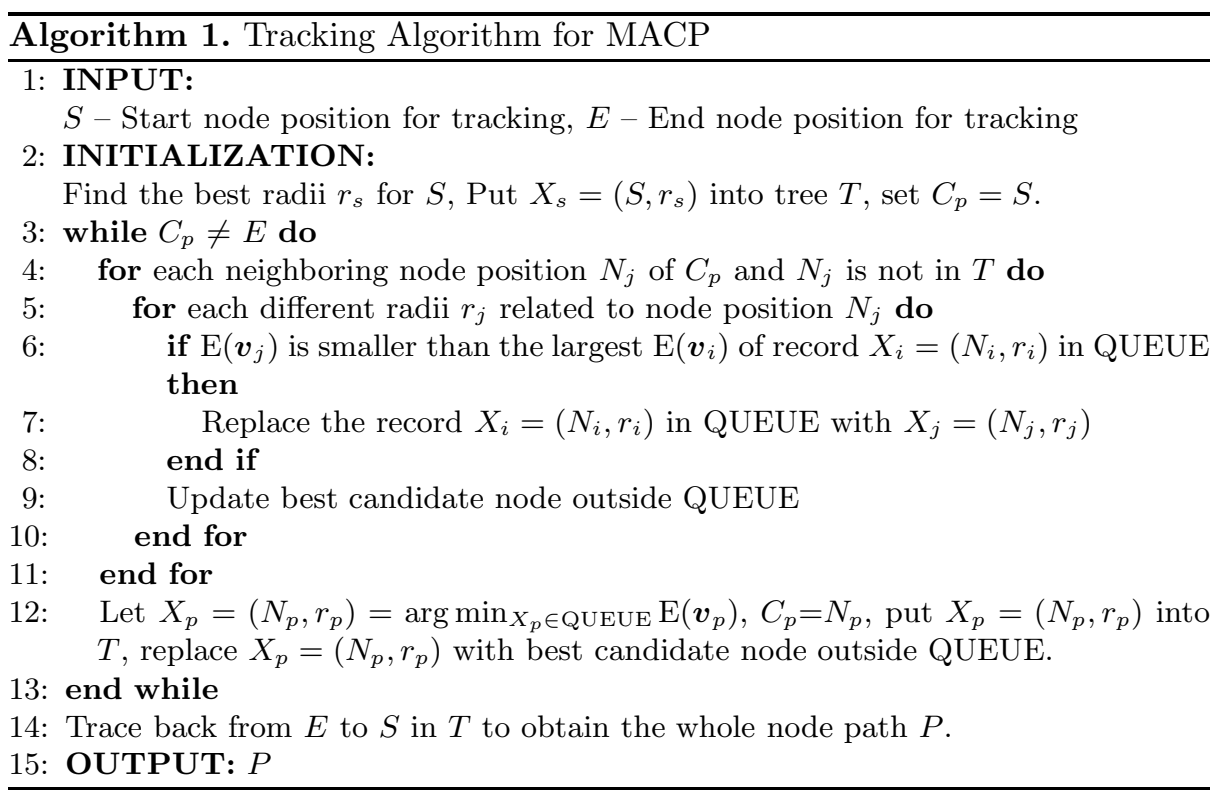

$T$. So the proposed method can trace the vessel with a lower computational cost than minimal path methods and realize the real time segmentation.

During the propagation, the energy term $\mathrm{E}\left(\boldsymbol{v}_{j}\right)$ in Algorithm 1 is calculated by Equation (4). The prior term $P\left(X_{t} \mid X_{t-1}\right)$ in constrained radius variations is described in [5]. Observations $Y_{t}$ are the responses of a multi-scale oriented medialness feature and details can be found in [6].

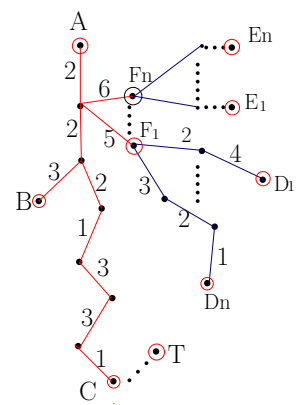

Fig. 3. Efficiency of MACP Model

\section{Experiment and Validation}

The centerlines of 24 testing datasets (96 vessels) were submitted to the Rotterdam Coronary Artery Algorithm Evaluation Framework [1] and the evaluation results are reported in Table 1. The overlap percentage between the experiment 
Table 1. Results from the Rotterdam Framework

\begin{tabular}{|c|l|l|l|l|l|l|}
\hline & \multicolumn{3}{|c|}{ \% / mm } & \multicolumn{3}{c|}{ score } \\
Measure & \multicolumn{1}{|c|}{ min. } & \multicolumn{1}{|c|}{ max. } & avg. & min. & max. & avg. \\
\hline OV & $60.8 \%$ & $100.0 \%$ & $93.4 \%$ & 34.3 & 100.0 & 66.6 \\
OT & $60.7 \%$ & $100.0 \%$ & $93.6 \%$ & 30.4 & 100.0 & 67.7 \\
AI & $0.24 \mathrm{~mm}$ & $0.70 \mathrm{~mm}$ & $0.41 \mathrm{~mm}$ & 17.5 & 54.2 & 28.3 \\
\hline
\end{tabular}

results and the ground truth is denoted as OV, which is the most important measure in the framework. OT gives an indication of how well the method is able to track the clinically relevant vessel section with a diameter of $1.5 \mathrm{~mm}$ or larger. AI denotes the accuracy of centerline extraction, provided that the evaluated centerline is inside the vessel. The score is defined between 0 and 100, with 50 for a result of the order of the inter-observer variability. We note that, $S_{p}, E_{p}$ and $A_{p}$ are provided by the Evaluation Framework.

The relatively good performance of our method was confirmed with $93.4 \%$ overlap percentage $(\mathrm{OV})$ on average, and the score for overlap percentage is 66.6, which means the proposed method exceeds inter-observer overlap with its score 50 . The radii of the typical coronaries range from $0.5 \mathrm{~mm}$ to $4.0 \mathrm{~mm}$, and the spacing of the images is about $0.3 \mathrm{~mm}$ to $0.4 \mathrm{~mm}$ in each direction, so the average result achieved $(0.41 \mathrm{~mm})$ for measure AI is fine. To improve the AI measure, we can perform interpolation to the image and obtain more node positions. However, it will be slow to trace the path in the interpolated image. Thus it is a trade-off between AI and computational efficiency. OF denotes how much a coronary artery has been extracted before making an error, but it is meaningless for the proposed method and we omit it here. In the proposed method, the tracking starts from point $A_{p}$ back to point $S_{p}$ and from point $A_{p}$ to $E_{p}$, so the measure $\mathrm{OF}$ actually cannot denote how much of a vessel has been extracted before making the first error.

To evaluate the computation time of the proposed method, we recorded the tracking time of the training datasets (32 vessels) with different $Q$ values, $Q$ is the size of QUEUE in Algorithm 1] As we can see in Table 2, the computation time increases monotonously with $Q$. For $Q=20$, with which we obtain the $93.4 \%$ on $\mathrm{OV}$, the average computation time is 7.467 seconds. According to 8], the reference standard centerlines are about $138 \mathrm{~mm}$ on average, so the speed of tracing is $18.5 \mathrm{~mm} / \mathrm{s}$. The processor used in the experiment is dual core AMD Opteron $2216(2.4 \mathrm{GHz})$ and the physical memory is $8 \mathrm{~GB}$. For running the program, the maximum memory used is $3 \mathrm{~GB}$ even though the raw image and the gradient vector field for the whole image are restored in the memory.

Table 2. Computation Time (in seconds) of MACP

\begin{tabular}{|c|lll|}
\hline Size & min. & max. & avg. \\
\hline $\mathrm{Q}=10$ & 1.943 & 11.242 & 5.804 \\
\hline $\mathrm{Q}=20$ & 2.015 & 16.137 & 7.467 \\
\hline $\mathrm{Q}=30$ & 2.058 & 18.945 & 7.953 \\
\hline Average & 2.005 & 15.441 & 7.075 \\
\hline
\end{tabular}


Given centerlines and related radii, surfaces can be obtained. In Fig. 4(a) surface was rendered from reference centerlines and radii from [1]. Surface for the proposed method is shown in Fig. 4(b). Since the centerlines obtained by the proposed method are based on voxels, the number of voxels on the centerlines obtained is much less than the reference. Also, radius $r_{t}$ associated with $\boldsymbol{p}_{t}$ ranges from $r_{t-1}-0.6 \mathrm{~mm}$ to $r_{t-1}+0.6 \mathrm{~mm}$ with a fixed step of $0.3 \mathrm{~mm}$. These all may make it difficult to have a smooth rendering surface. However, the rendering surface for our method is satisfactory.

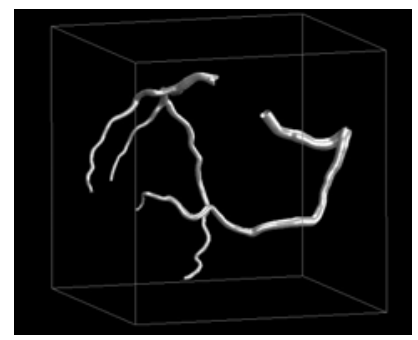

(a) Surface for Ground Truth

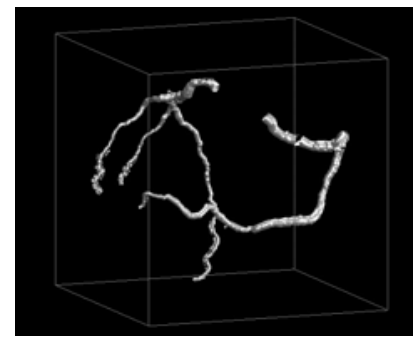

(b) Surface for Our Method

Fig. 4. Rendering Surface Results

Table 3 presents the experimental results of 15 state-of-the-art approaches in the Rotterdam evaluation framework [1]. As we can see in Table 3, the computational efficiency (7.467 seconds per segment, 29.868 seconds per dataset) of our method is better than all approaches. The only one approach [9], with its computation time comparable with the proposed method, however achieves a lower overlap performance $\mathrm{OV}(84.7 \%)$.

There are only four [5]3]10 2] out of fifteen approaches having better overlapping performance than the proposed method (93.4\%). However, the proposed method is much faster than all of them. Meanwhile, these methods have other problems. In Friman's MHT method [3], more human intervene is required to see whether the tracking is finished. In [10, vessel enhancement is performed

Table 3. Experimental Results From [1]

\begin{tabular}{|c|c|c|}
\hline Measure & OV & Computation Time \\
\hline MHT(Friman et al., 2008) 3$]$ & $98.5 \%$ & 6 minutes \\
\hline BayesianMaxPaths (Lesage, 2008) [5] & $97.5 \%$ & 4 minutes \\
\hline $\begin{array}{lll}\text { VirtualContrast2b(Wang et al., 2008) } & 10\end{array}$ & $96.7 \%$ & 2 minutes \\
\hline $\begin{array}{lll}\text { Tracer }(\text { Szymczak, 2008) } & 2 \\
\end{array}$ & $95.1 \%$ & 30 minutes \\
\hline GVFTubenLinkage (Bauer and Bischof, 2008) & $92.7 \%$ & 10 minutes \\
\hline TwoPointMinCost(Metz et al., 2008) & $91.9 \%$ & 12 minutes \\
\hline AxialSymmetry(Dikici et al., 2008) & $90.8 \%$ & 5 minutes \\
\hline 3DInteractiveTrack(Zhang et al., 2008) & $89.6 \%$ & $3-6$ minutes \\
\hline KnowledgeBasedMinPath (Krissian et al., 2008) & $88.0 \%$ & 7 hours \\
\hline AutoCoronaryTree (Tek et al., 2008) 9 & $84.7 \%$ & $<30$ senconds \\
\hline DepthFirstModelFit (Zambal et al., 2008) & $84.7 \%$ & $4-8$ minutes \\
\hline CocomoBeach (Kitslaar et al., 2008) & $78.8 \%$ & 70 seconds \\
\hline ElasticModel(Hernndez Hoyos et al.) & $77.0 \%$ & $2-6$ minites \\
\hline VirtualContrast(Zhang et al.) & $75.6 \%$ & 2 minites \\
\hline CoronaryTreeMorphoRec (Castro et al., 2008) & $67.0 \%$ & 30 minutes \\
\hline
\end{tabular}


before tracking. Actually, it is impossible for the methods like [10] to run in real time if enhancement process is required since it is a time consuming process. The computational cost of 2 is much higher than the proposed method, however, the superiority of OV of the method is not so obvious (95.1\% vs $93.4 \%$ ). In contrast with the proposed method, 3[10]2 do not estimate the vessel radii and this leads to difficulty in rendering the vessel surfaces as we can have in Fig. 4(b) Although the overlap performance (OV) of methods [5/3] is high, the use of minimal path methods may lead to shortcut problem in some cases as shown in Fig. 1.

\section{Conclusion}

In this paper, we have proposed a MACP model for 3D coronary artery segmentation and presented the use of Prim's Minimum Spanning Tree method for efficient optimization of the MACP model. The centerline and the radii of the cross sections of the coronary artery are extracted simultaneously during the optimization on the discrete $4 \mathrm{D}$ graph. The method has been validated intensively with Rotterdam Coronary Artery Algorithm Evaluation Framework [1] and the tracking quality is good, and it does not need any image preprocessing steps. The computational cost is particularly low, and this makes real time segmentation of coronary artery possible. Shortcut problem, which is a classic issue of the minimal path techniques, can be overcome by the proposed method.

\section{References}

1. Rotterdam Coronary Artery Algorithm Evaluation Framework, http://coronary.bigr.nl/

2. Vessel tracking by connecting the dots, http://hdl.handle.net/10380/1406

3. Friman, O., Kühnel, C., Peitgen, H.: Coronary artery centerline extraction using multiple hypothesis tracking and minimal paths. The MIDAS Journal (2008)

4. Lesage, D., Angelini, E., Bloch, I., Funka-Lea, G.: A review of 3D vessel lumen segmentation techniques: Models, features and extraction schemes. Medical Image Analysis 13(6), 819-845 (2009)

5. Lesage, D., Angelini, E., Bloch, I., Funka-Lea, G.: Bayesian maximal paths for coronary artery segmentation from 3D CT angiograms. In: Yang, G.-Z., Hawkes, D., Rueckert, D., Noble, A., Taylor, C. (eds.) MICCAI 2009. LNCS, vol. 5761, pp. 222-229. Springer, Heidelberg (2009)

6. Lesage, D., Angelini, E., Bloch, I., Funka-Lea, G.: Design and study of flux-based features for 3D vascular tracking. In: ISBI. pp. 226-229 (2009)

7. Li, H., Yezzi, A.: Vessels as 4-D curves: Global minimal 4-D paths to extract 3-D tubular surfaces and centerlines. IEEE TMI 26(9), 1213 (2007)

8. Schaap, M., Metz, C., Walsum, T.v., et al.: Standardized evaluation methodology and reference database for evaluating coronary artery centerline extraction algorithms. Medical Image Analysis 13(5), 701-714 (2009) 
9. Tek, H., Gulsun, M., Laguitton, S., Grady, L., Lesage, D., Funka-Lea, G.: Automatic coronary tree modeling. The MIDAS Journal (2008)

10. Wang, C., Smedby, Ö.: Integrating automatic and interactive methods for coronary artery segmentation: let the pacs workstation think ahead. International Journal of Computer Assisted Radiology and Surgery, 1-11

11. Wink, O., Niessen, W., Viergever, M.: Multiscale vessel tracking. IEEE TMI 23(1), 130-133 (2004) 